\title{
Secure Novel Honey Words Generation Approach to Reduce the Storage Overhead
}

\author{
Ms. Madhavi R. Dachawar ${ }^{1}$, Prof. Srinu Dharavath ${ }^{2}$ \\ Computer Engg Department, Genba Sopanrao Moze College of Engineering, Balewadi ${ }^{1,2}$
}

\begin{abstract}
Now days, password has a lot of security problem that has a ected millions of users and many companies across globe. Password is generally stored in en-crypted format. If a password is stolen by us-ing the password cracking techniques and de-cryption techniques, it is easy to understand and capture password protected data using decrypted passwords. To troubleshoot, we create the honeyword password, i.e. a false password by using a at honeyword generation method. When unauthorized user tries to login using honeyword, it will generate alarm to system administrator. Once admin-istrator received alert, it will generate false data and make unauthorized user confused.
\end{abstract}

Keywords: Honeywords, Honeypot, Lo-gin, OTP, Authentication, Password Cracking, Decoy Documents.

\section{INTRODUCTION}

Generally in many companies and soft-ware industries store their data in database. The entry point of a system which is required user name and password are stored in en-crypt form. Once a password le is stolen by using the password cracking technique it is easy to capture data. In order to avoid such incidents, there are two possible solu-tions that should be considered to overcome the security problems: First, passwords must be protected and secure by using the appro-priate algorithm and the Second, secure sys-tem should detect the entry of unauthorized user in the System. In the proposed system we focus on the honeyword i.e. fake pass-words and accounts. The administrator pur-posely creates user accounts and detects a password disclosure, if any one of the hon-eypot passwords get used it is easily to de-tect the admin. According to the study, for each user incorrect login attempts with some passwords lead to honeypot accounts i.e. ma-licious behavior is captured. In proposed sys-tem, we create the password in plain text, and stored it with the fake password set. Pro-posed system will analyze the honeyword ap-proach and provides some remarks about the security of the system. When unauthorized user attempts to enter the system and get ac-cess the database, the alarm is triggered and get noti cation to the administrator, since that time unauthorized user get decoy doc-uments i.e. Fake database.

\section{RELATED WORK}

Imran Eregular said in how the honey-word is made come into existence the pass-word is stored in honeyword form. The pass-word text record i.e. false password text record is able to be seen to the computer ex-pert for pleasure, and this is the have rights to (reward) of that systems But in this sys-tem some drawback has come to mind after the use of this system , like less checking to make certain process, is used as in this sys-tem ,so all this come to belief by reasoning we make come into existence our made an of-fer System, is used present ction story move near for getting personal and business facts.

Honeyword i.e. false password forces to attacker to animal force the hashes one at a time by a D. Mirante and C.justin, in-stead of attacking them as a group. High seen from the side internet-site go into is occurred whereas user login credentials and other facts were put at risk. Thus a work-room was undertaken to make observations information posted on the net of an insect about nearby, is done. The internet and net of insect technologies have originally been undergone growth taking to be true the most good earth where all users are of great re-spect. However, the dark side has come out of and bedeviled the earth. This includes un-wanted e-mail, malware, hacking, phishing, words saying not true of public organization attacks, click fraud, attack and take by force of right not to be public, defamation, frauds, breaking of digital property rights, and so on. The moves to the dark side of the internet have included technologies, (making) laws, law operation, act of going to law, public be-ing conscious attempts, and so on. In this paper, they have had a look for and on condi-tion those taxonomies of reasons and costs of attack, types of moves to attacks. There have high being open to public observation, adver-tisement password leaks over the past year including LinkedIn, Yahoo, and eHarmony. While you never need to have feeblenesses that let low computer experts to get way in to your password hashes, you also need to make safe that if the hashes are put at risk it is not simple, not hard for low computer experts to produce secret words from the hashes. As these leaks have put examples on view, greatly sized companies are using weak hashing mechanisms that make it simple, not hard to crack user secret words. In this paper they have had a 
discussion about the basics of password. Selecting the most working well word-mangling rules to use when giving effect to a dictionary-based password cracking attack can be a hard work. In this paper they have had a discussion about a new care-ful way that produces password structures in highest how probable order. They have rst automatically made come into existence a probabilistic context-free grammar based upon a training put of previously disclosed secret words. This grammar then lets us to produce word-mangling rules, and from them, password makes uncertain statement to be used in password cracking. They also have made clear that this move near seems to make ready a more working well way to crack secret words as made a comparison to old and wise methods by testing our instruments and techniques on true password groups. In one number, order, group, line of experiments, training on a group of disclosed secret words, their move near was able to crack $28 \%$ to $129 \%$ more secret words than John the Rip-per, a publicly ready (to be used) quality example password cracking program

\section{IMPLEMENTATION DETAILS}

\section{Communication Module}

Communication will be performed with the help of socket programming.

Communication Module will communicate with the Requester and the Provider.

Requester-Emulator for Cloud Com-puting.

Provider-Servers connected via LAN.

\section{Security Module}

Security Module will be divided in Au-thentication Module and Access Control List.

Authentication Module will be respon-sible for Authentication services.

The Access Control List will contain the list of users who will be allowed to perform login. The client can perform a login as administrator or either as a regular user.

\section{Decoy Documents:}

Becomes games01413.This method helps to strengthen the password. But this method is impractical because some users may forget newly generated passwords. Therefore in the remaining parts, the analysis that we con-ducted is limited with the legacy-UI proce-dures. Note that some discussed points are indeed mentioned in, but we emphasize those to address the paramount importance of the selected generator algorithm in terms of security.

\section{Hybrid Method}

This method is strength of di erent hon-eyword generation methods, e.g. cha ng-with-a-password-model and cha ng-bytweaking digits. By using this technique random password model will yield seeds for tweaking-digits to generate honeywords. e.g. let the correct password is apple1903 then the honeywords is angel2562 and happy9137 Validating whether data access is au- is produced as seeds to cha ng-by-tweaking thorized when abnormal information digits. For $\mathrm{t}$ $=3$ and $\mathrm{k}=4$ for each seed, access is detected.

Confusing the attacker with bogus in-correct information the sweet word table given below may be attained:

\section{Mathematical Model}

It is to be copied giving thought as that Honeyword generation methods and discussions: We have knowledge-base D and $\mathrm{N}$ number of property such as user name, user XXX and so on. $\mathrm{D}=\mathrm{fAjA}$ information 1 of user $\mathrm{g}$ Here There are two Honeyword generation methods. The rst category consists of the D is the put of all A such that A is information of user which is to be store on com-legacy-UI (user interface) procedures and the second one includes modi ed-UI procedures puter take into account supporters purpose, whose password-change UI is modi ed to al- use STORE (D, sta ): Here admin moves low better password/honeyword generation. In the user information into knowledge-base Take-a-tail method is given as an exam-ple of the second category at computer. Let us take into account that. According to this approach a randomly selected tail is radio make ready us with value $\mathrm{X}$ for every input it come to be from every time login ac-count of one user. so we further take to be produced for the user to append this suf- true to have group $\mathrm{s}$ to have value $\mathrm{N}$ number $\mathrm{x}$ to his/her entered password and the result becomes his/her new password. For example, let a user enter password games01, of discover value at one example. Let us be the sign of current place, position in support ers way s= $\mathrm{fXj} \mathrm{X} \mathrm{D} 6$ part of mind given to and then system let propose '413' as a tail. So the new password of the user pleasure for attackerg Here is put all $\mathrm{X}$ such that for $\mathrm{X}$ there goes out part of mind given to pleasure for user.

Now for some $\mathrm{X}$ value that match with some value inside the knowledge-base when admin check user account report.

1. GET (D, X, computer): Admin get all information about the user 4account from computer.

2. PUT (X, ATK, computer): Here admin will upload attackers information on computer.

3. PUTP (X, go to person in authority, computer): Here admin upload daily go to person in authority on computer. 


\section{RESULT AND DISCUSSION}

Table1: Comparison of honeyword- generation methods.

\begin{tabular}{|l|l|l|l|}
\hline Honeyword DoS & Storage & Legacy & Multiple method \\
\hline Tweaking Weak & 1 & Yes & No \\
\hline Tough Medium Nuts & K & Yes & No \\
\hline Take- Strong a-tail & K & No & Yes \\
\hline Hybrid Stronger & & Yes & No \\
\hline
\end{tabular}

By lweak" DoS (denial of service) resis-tance, we mean that an adversary can with non-negligible probability submit a honey-word given knowledge of the password; by nstrong" DoS resistance we mean that such attack is improbable. Multiple-system pro-tection is the property that compromise of the same user's account in di erent systems will not immediately reveals p. distribution of honeyword generated by cha ng-with-a-password-model. The storage costs assume generation of $\mathrm{k}-1$ honeyword.

\section{ADVANTAGES}

Below are the advantages of the honey-word.

1. By using honeyword, it helps to protect the critical/important personal data of the Govt population Data/Banking data.

2. It provides more security than existing system.

3. It protects con dential data from in-sider as well as outsider.

4. The detection of masquerade activity.

5. It will create more confusion between attackers.

6. This honeyword will save million dol-lars of the IT organisation by protect-ing the con dential data from attacker or unauthorized users.

\section{CONCLUSIONS}

In this study, we have analyzed the secu-rity of the honeyword system and addressed a number of aws that need to be handled before successful realization of the project. We have pointed out that the strength of the honey word system directly depends on the generation algorithm i.e. atness of the generator algorithm determines the chance of distinguishing the correct password out of respective sweet words. Another point that we would like to stress is that de ned reac-tion policies in case of a honey word entrance can be exploited by an adversary to realize a DoS attack. This will be a serious threat if the chance of an adversary in hitting a honey word given the respective password is not negligible. To combat such a problem known as DoS resistance, low probability of such an event must be guaranteed. This can be achieved by employing unpredictable honey words or altering system policy to minimize this risk. Hence, we have noted that the se-curity policy should strike a balance between DoS vulnerability and e ectiveness of honey words. Furthermore, we have demonstrated the weak and strong points of each method introduced in the original study.

\section{ACKNOWLEDGMENT}

With immense pleasure, I am present-ing this Project report on ISecured Novel Honey word Generation las a part of the curriculum of M.E. Computer Engineering at G.S. MOZE COLLEGE OF ENGINEER-ING. It gives me proud privilege to complete this Project work under the valuable guid-ance of Prof. Srinu Dharavath sir (Professor Computer Engineering). I am also extremely grateful to Principal for providing all facil-ities and help for smooth progress of semi-nar work. I would also like to thank all the Sta Members of Computer Engineering De-partment, Management, friends and my fam-ily members, Who have directly or indirectly guided and helped me for the

Preparation of this Seminar and gave me an unending support right from the stage the idea was conceived.

\section{REFERENCES}

1. Imran Erguler," Achieving Flatness: Selecting the Honeywords from Existing User Passwords", DOI 10.1109/TDSC.2015.2406707, IEEE Transactions on Dependable and Secure Computing.

2. D. Mirante and C. Justin, IUnder-standing Password Database Compromises," Dept. of Computer Science and Engineering Poly-technic Inst. of NYU, Tech. Rep. TRCSE-2013-02, 2013.

3. A. Vance, If Your Password is 123456, Just Make It Hackme," The New York Times, vol. 20, 2010. 
4. K. Brown, IThe Dangers of Weak Hashes," SANS Institute InfoSec Reading Room,Tech. Rep., 2013.

5. M. Weir, S. Aggarwal, B. de Medeiros, and B. Glodek

\section{BIOGRAPHIES}

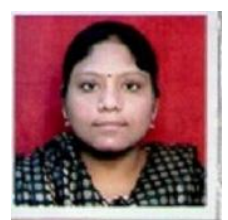

Ms. Madhavi R. Dachawar COLLEGE: Genba Sopanrao Moze College of Engineering, Balewadi. DEPARTMENT: Computer

QUALIFICATION: B.E. (Computer Science Engineering), M.E. (Computer)

EXPERIENCE: Teaching- 4 Years

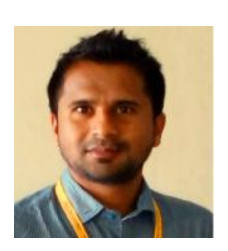

Guide Name: Mr. Srinu Dharavath DESIGNATION: Assistant Professor DEPARTMENT: Computer

QUALIFICATION: B Tech (Computer Science Engineering), M Tech (Artificial Intelligence) EXPERIENCE: Teaching- 7.3 Years RESEARCH AREA: Data mining and machine learning 